\title{
Complications post-opératoires et mode de prise en charge en angioplastie : apport du Programme de Médicalisation des Systèmes d'Information (PMSI)
}

\author{
Yann Rivault ${ }^{* 1,2}$, Nolwenn Le Meur ${ }^{1}$, and Olivier Dameron ${ }^{2}$ \\ ${ }^{1}$ École des hautes études en santé publique [Rennes] (EHESP) - PRES Sorbonne Paris Cité, PRES \\ Université Européenne de Bretagne (UEB) - Avenue du Professeur Léon-Bernard - CS 74312 - 35043 \\ Rennes cedex, France \\ ${ }^{2}$ DYLISS (INRIA - IRISA) - INRIA, Universite de Rennes 1, CNRS : UMR6074 - Campus de Beaulieu \\ 35042 Rennes cedex, France
}

\section{Résumé}

Introduction

La chirurgie ambulatoire est un mode de prise en charge permettant de raccourcir la durée d'hospitalisation à une seule journée. Elle connaît un réel développement en angioplastie. Néanmoins, comme toute chirurgie, elle n'est pas exempte de complications.

Nous avons mené une étude pilote pour étudier la pertinence du PMSI pour la recherche des facteurs déterminants d'une complication post-chirurgie.

\section{Matériel et Méthodes}

La population était constituée de 4889 séjours hospitaliers pour angioplastie, extraits du PMSI pour les années 2012 et 2013. Les variables étudiées étaient des variables individuelles et d'hôpital, telles que l'âge et le sexe des patients, le mode de prise en charge ou encore un indice de comorbidité.

L'analyse statistique a été réalisée grâce à une méthode de régression logistique multiniveaux. Elle a permis d'ajouter des effets aléatoires sur les variables étudiées, grâce à l'introduction au modèle d'un second niveau, indexé sur la région de résidence des patients opérés, pour prendre en compte une dimension géographique.

\section{Résultats}

Le risque estimé de développer une complication suite à une opération chirurgicale d'angioplastie par le modèle retenu s'explique grâce au score de comorbidité et au mode de prise en charge. Ce modèle autorise les coefficients associés au facteur du mode de prise en charge à varier d'une région à l'autre. Excepté une seule région, celle des Midi-Pyrénées, le risque estimé de complication semble plus élevé pour un séjour se déroulant en ambulatoire.

Conclusion

\footnotetext{
*Intervenant
} 
La méthode statistique mise en place a permis de mettre en évidence l'effet de certains facteurs sur le risque estimé de complication, notamment le facteur du mode de prise en charge, et les disparités que pouvait avoir cet effet d'une région à l'autre.

Nous envisageons d'améliorer cette étude par l'élaboration d'indicateurs de déviance des parcours de soins, à étudier comme de potentiels facteurs déterminants d'une complication.

Mots-Clés: ambulatoire, complications post, opératoire, angioplastie, bases médico, administratives 